\title{
Sífilis na gestação: barreiras na assistência pré-natal para o controle da transmissão vertical
}

\section{Syphilis in gestation: barriers in prenatal care for the control of vertical transmission}

\author{
Vilma Costa de Macêdo' (D), Luciana Maria Delgado Romaguera² (D), \\ Mariana Oliveira de Alencar Ramalho ${ }^{3}$ (D) , Lygia Carmen de Moraes Vanderlei ${ }^{4}$ (D), \\ Paulo Germano de Frias ${ }^{5}$ (D) Pedro Israel Cabral de Lira ${ }^{6}$ (D) \\ 'Departamento de Enfermagem, Universidade Federal de Pernambuco (UFPE) - Recife (PE), Brasil. \\ ${ }^{2}$ Unidade Neonatal do Hospital das Clínicas, Universidade Federal de Pernambuco (UFPE) - Recife (PE), Brasil. \\ ${ }^{3}$ Programa de Pós-graduação em Saúde da Criança e do Adolescente, Universidade Federal de Pernambuco (UFPE) - Recife (PE), \\ Brasil. \\ ${ }^{4}$ Diretoria de ensino, Instituto de Medicina Integral Professor Fernando Figueira (IMIP) - Recife (PE), Brasil. \\ ${ }^{5}$ Grupo de Estudos em Avaliação em Saúde, Instituto de Medicina Integral Professor Fernando Figueira (IMIP) -Recife (PE), Brasil. \\ ${ }^{6}$ Departamento de Nutrição, Universidade Federal de Pernambuco (UFPE) - Recife (PE), Brasil.
}

Como citar: Macêdo VC, Romaguera LMD, Ramalho MOA, Vanderlei LCM, Frias PG, Lira PIC. Sífilis na gestação: barreiras na assistência pré-natal para o controle da transmissão vertical. Cad Saúde Colet, 2020;28(4):518-528.

https://doi.org/10.1590/1414-462X202028040395

\section{Resumo}

Introdução: A sífilis gestacional é uma doença de transmissão vertical, da mãe para o feto, que se não tratada, pode resultar em inúmeros desfechos negativos para a saúde materna e infantil. Objetivo: Avaliar as barreiras na assistência pré-natal para o controle da transmissão vertical da sífilis em gestantes segundo o perfil sociodemográfico, reprodutivo e assistencial em uma metrópole do Nordeste brasileiro. Método: Estudo descritivo, conduzido a partir de banco de dados de um estudo caso-controle para sífilis gestacional em maternidades públicas no Nordeste do Brasil, entre 2013 e 2014. As informações do acompanhamento e tratamento foram obtidas pelos registros do cartão do pré-natal e entrevistas. O diagnóstico de sífilis considerou os registros do cartão, anotações em prontuário e resultados do Venereal Disease Research Laboratory (VDRL). Resultados: Foram incluídas 1.206 mulheres, 91,7\% realizaram pré-natal e se declararam, em maior proporção, como casadas, menor número de filhos e maior escolaridade. O resultado do VDRL do pré-natal foi anotado em 23,9\%. Entre as 838 mulheres que receberam o VDRL no pré-natal, 21\% eram reagentes e 70,5\% trataram a infecção. Destas, 69,4\% utilizaram o esquema para sífilis terciária e $8,1 \%$ trataram com outras medicações. Conclusão: O pré-natal não alcançou a efetividade na prevenção e rastreio da sífilis, uma vez que ocorreram mulheres reagentes para a infecção na admissão à maternidade, ainda que em menor proporção, sendo perdida a oportunidade de alcançar o controle da doença.

Palavras-chave: sífilis; gestantes; sífilis congênita; cuidado pré-natal; maternidades.

\section{Abstract}

Background: Gestational syphilis is a mother-to-fetus mother-to-child disease that, if left untreated, can result in numerous negative outcomes for maternal and child health. Objective: Evaluate the barriers in prenatal care for the control of vertical transmission of syphilis in pregnant women according to the sociodemographic, reproductive, and care profile in a metropolis of northeastern Brazil. Method: This is a descriptive study, conducted from a database of a case-control study for gestational syphilis in

Trabalho realizado em sete maternidades públicas localizadas no município do Recife -PE com diferentes níveis de gestão e realizam atendimento à mulher durante o período gestacional exclusivamente pelo SUS: Instituto de Medicina Integral Prof. Fernando Figueira (IMIP) Hospital Agamenon Magalhães (HAM) Hospital Barão de Lucena (HBL) Hospital das Clínicas (HC) Policlínica e Maternidade Arnaldo Marques Maternidade Professor Bandeira Filho Unidade Mista Professor Barros Lima - Recife (PE), Brasil.

Correspondência:Vilma Costa de Macêdo. E-mail: vilma.macedo@ufpe.br

Este é um artigo publicado em acesso aberto (Open Access) sob a licença Creative Commons Attribution, que permite uso, distribuição e reprodução em qualquer meio, sem restrições desde que o trabalho original seja corretamente citado. 
public maternity hospitals in the Northeast of Brazil, between 2013 and 2014. Information on follow-up and treatment was obtained through prenatal card records and interviews. The diagnosis of syphilis considered card records, chart notes, and results from the Venereal Disease Research Laboratory (VDRL). Results: A total of 1,206 women were included, 91.7\% were prenatal and declared to be married, with fewer children and higher educational levels. The VDRL result of prenatal care was noted at $23.9 \%$. Among the 838 women who received the VDRL in prenatal care, $21 \%$ were reactants and $70.5 \%$ treated the infection. Of these, $69.4 \%$ used the scheme for tertiary syphilis and $8.1 \%$ were treated with other medications. Conclusion: Prenatal care did not reach effectiveness in the prevention and screening of syphilis since women reacting to the infection on maternity admission occurred, albeit to a lesser extent, and the opportunity to achieve control of the disease was lost.

Keywords: syphilis; pregnant women; syphilis congenital; prenatal care; maternity hospitals.

\section{INTRODUÇÃO}

Mundialmente, cerca de 1,9 milhão de gestantes foram infectadas pela sífilis em 2013, principalmente nos países em desenvolvimento, revelando a magnitude de um importante

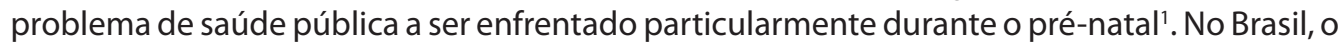
último estudo sobre gestantes com sífilis em serviços de saúde públicos e privados, realizado em 2012, estimou uma prevalência de 1,02\% $\%^{2}$. Nos anos subsequentes, ocorreu aumento das notificações da sífilis gestacional, apesar de persistirem maiores registros de casos de sífilis congênita ${ }^{3}$.

De forma geral, tanto a sífilis gestacional quanto a congênita apresentam elevado sub-registro, no Brasil e estados além de outros países da América Latina e Caribe ${ }^{4}$. Em 2017, foram notificados no Brasil 49.013 casos de gestantes com a infecção, conferindo uma taxa de detecção de 17,2 por 1.000 nascidos vivos. Apesar da melhoria da notificação entre as regiões brasileiras, persistem diferenciais na magnitude da doença e na capacidade de realizar a vigilância e análise confiável do problema entre os estados ${ }^{3,5}$. A taxa de transmissão vertical da sífilis gestacional no país entre os anos de 2011 e 2012 foi de 34,3\%, variando de 37,9\% na região Nordeste a $15 \%$ no Centro-Oeste 6 .

A sífilis gestacional agrega o risco de transmissão vertical e, quando não tratada, cerca de $40 \%$ dos casos resultam em desfechos negativos, relacionados ao aborto espontâneo, morte fetal ou neonatal precoce ou ainda graves sequelas perinatais ${ }^{7}$. O pré-natal é o único momento possível para identificação e redução dos riscos, considerando a triagem sorológica e o tratamento adequado da gestante e parceiros ${ }^{8,9}$. A despeito da elevada cobertura da assistência pré-natal alcançada no Brasil, permanecem barreiras para o acesso oportuno das gestantes, evidenciando dificuldades na superação de desigualdades sociais, especialmente entre as mais vulneráveis: indígenas, pretas, de menor escolaridade, com maior número de gestações e as residentes nas regiões Norte e Nordeste ${ }^{10}$.

Adicionalmente, mesmo entre as mulheres que realizaram o pré-natal, muitas são as oportunidades perdidas no diagnóstico e tratamento dos casos. A remoção de obstáculos para realização de um pré-natal adequado envolve, entre outras questões, a captação precoce da gestante, o aconselhamento, a intervenção educacional, a solicitação e realização dos exames conforme protocolos, o recebimento de resultados em tempo oportuno, além do tratamento adequado da mulher e seu parceiro ${ }^{2,11-13}$.

Muitas das deficiências assistenciais durante o pré-natal podem ser constatadas apenas no momento da admissão à maternidade. Nessa ocasião ocorre a última oportunidade diagnóstica da sífilis para a gestante e parturiente, porém mantido o risco da infecção congênita ${ }^{2,5,8}$.

O conhecimento sobre a realização, adequação e oportunidade do pré-natal e do tratamento adequado para sífilis no contexto sociodemográfico e assistencial de mulheres pode contribuir para o estabelecimento de estratégias para o seu enfrentamento. Estas, para serem efetivas, devem ser direcionadas à promoção da saúde, prevenção dos potenciais fatores de risco, diagnóstico e tratamento, especialmente entre os grupos mais expostos à infecção ${ }^{14,15}$.

O objetivo deste estudo foi avaliar as barreiras na assistência pré-natal para o controle da transmissão vertical da sífilis em gestantes segundo o perfil sociodemográfico, reprodutivo e assistencial em uma metrópole do Nordeste brasileiro. 


\section{MÉTODO}

A partir de banco de dados de um estudo de caso-controle ${ }^{15}$, realizado entre 2013 e 2014 para determinar os fatores de risco na ocorrência de sífilis em gestantes, avaliaram-se transversalmente as ações relacionadas ao controle da transmissão vertical da infecção no pré-natal.

As informações referem-se às 1.206 mulheres admitidas em sete maternidades do SUS Recife, Pernambuco, em trabalho de parto, pós-parto, aborto ou intercorrências clínicas e cirúrgicas da gravidez ou puerpério, após o resultado do exame Venereal Disease Research Laboratory (VDRL) sob qualquer titulação. Realizou-se entrevista estruturada, consulta ao cartão de pré-natal, ficha de admissão, prontuário clínico (transcrição de datas, resultado do VDRL e tratamento), livros/sistemas informatizados laboratoriais para os resultados de exames de admissão.

Utilizaram-se variáveis demográficas (idade, cor da pele autodeclarada, estado civil e local de residência), sociais (escolaridade, classe econômica, conforme critério da Associação Brasileira de Empresas de Pesquisa - Abep), saúde sexual e reprodutiva (idade na primeira relação sexual e na primeira gravidez, número de gestações anteriores e de parceiros sexuais e aborto anterior) e assistenciais (local do pré-natal, número de consultas, trimestre de início, solicitação de exames, entrega de resultados e categoria profissional que o realizou). A definição de tratamento adequado considerou: utilizar a penicilina benzatina em doses preconizadas; finalizar o tratamento até trinta dias antes do parto e tratar concomitantemente o parceiro com o mesmo esquema.

Foram comparadas a condição de realização do pré-natal em relação às variáveis demográficas, sociais e da saúde sexual e reprodutiva e quanto ao registro do VDRL no cartão de pré-natal, as variáveis assistenciais. Realizaram-se análises univariadas com as variáveis: pré-natal (sim e não), VDRL no cartão do pré-natal/prontuário (registrado e não registrado), mulheres com VDRL reagentes no pré-natal (tratou e não tratou) associadas às características sociodemográficas, reprodutivas e assistenciais, sendo utilizado o teste do qui-quadrado $\left(X^{2}\right)$ para detectar diferenças entre as proporções com valores significantes de $p<0,05$.

O estudo foi submetido ao Comitê de Ética em Pesquisa em Seres Humanos da Universidade Federal de Pernambuco (UFPE), sendo aprovado de acordo com CAAE: 07353712.0.0000.5208.

\section{RESULTADOS}

Das 1.206 mulheres estudadas, 48,6\% residiam em Recife, 63,3\% tinham entre 20 e 34 anos, $65 \%$ se autodeclararam pardas e $61,4 \%$ em união estável, enquanto $8 \%$ eram analfabetas e $30 \%$ pertenciam às classes econômicas $\mathrm{D}$ e $\mathrm{E}$.

A não realização do pré-natal foi observada em $8,7 \%$ da amostra, e as mulheres, na maior parte, residiam na capital (12,8\%), se declararam solteiras, separadas ou viúvas $(15,4 \%)$, tinham menor escolaridade (22\%), com diferença estatisticamente significante em relação a ter realizado o pré-natal. Quanto aos antecedentes reprodutivos, predominaram as que relataram possuir três ou mais parceiros sexuais no último ano $(23,2 \%)$, aborto anterior $(17,2 \%)$, ter iniciado a primeira relação sexual e gravidez com quatorze anos ou menos $(11,4 \%$ e $14,7 \%$, respectivamente) (Tabela 1 ).

A análise do registro do VDRL no cartão do pré-natal ou prontuário mostrou que $23,9 \%$ não tinham informação e, entre estas, 57,6\% tiveram menor número de consultas e 57,1\% iniciaram o pré-natal no último trimestre. Entre as mulheres em que não foram solicitados exames na primeira consulta, 45,6\% não tinham registro de VDRL no cartão e, entre aquelas em que o exame foi solicitado e não havia registro, apenas $17,8 \%$ receberam os resultados em até quinze dias. Esses resultados apresentaram significância estatística (Tabela 2).

Avaliando os resultados das 52 gestantes que não trataram sífilis no pré-natal, $57,7 \%$ iniciaram no primeiro trimestre; $61,5 \%$ o realizaram nas unidades de saúde da família; $46,2 \%$ tiveram seis ou mais consultas, com solicitação de VDRL na primeira consulta em $92,3 \%$, e para $61,5 \%$ os resultados foram entregues em até quinze dias (Figura 1). 
Tabela 1. Características demográficas, sociais, reprodutivas das mulheres segundo assistência pré-natal na admissão à maternidade. Recife, Pernambuco, Brasil, 2013-2014

\begin{tabular}{|c|c|c|c|c|c|c|}
\hline \multirow{3}{*}{ Características } & \multirow{3}{*}{$\begin{array}{c}\text { Total } \\
n=1.206\end{array}$} & \multicolumn{4}{|c|}{ Pré-natal } & \multirow{3}{*}{ Valor $\mathbf{p}^{\mathrm{a}}$} \\
\hline & & \multicolumn{2}{|c|}{$\operatorname{Sim}(91,3 \%)$} & \multicolumn{2}{|c|}{ Não $(8,7 \%)$} & \\
\hline & & $n=1.101$ & $\%$ & $n=105$ & $\%$ & \\
\hline \multicolumn{7}{|l|}{ Local de residência } \\
\hline Região Metropolitana do Recife & 380 & 360 & 94,7 & 20 & 5,3 & \\
\hline Outros municípios & 240 & 230 & 95,8 & 10 & 4,2 & \\
\hline \multicolumn{7}{|l|}{ Idade (anos) } \\
\hline$\geq 35$ & 123 & 111 & 90,2 & 12 & 9,8 & 0,752 \\
\hline 20 a 34 & 764 & 701 & 91,8 & 63 & 8,3 & \\
\hline 12 a 19 & 319 & 289 & 90,6 & 30 & 9,4 & \\
\hline \multicolumn{7}{|l|}{ Cor da pele (autodeclarada) } \\
\hline Branca & 214 & 195 & 91,1 & 19 & 8,9 & 0,973 \\
\hline Preta & 152 & 138 & 90,8 & 14 & 9,2 & \\
\hline Parda & 784 & 716 & 91,3 & 68 & 8,7 & \\
\hline Amarela/indígena & 56 & 52 & 92,9 & 4 & 7,1 & \\
\hline \multicolumn{7}{|l|}{ Estado civil } \\
\hline Casada & 159 & 150 & 94,3 & 9 & 5,7 & $<0,001$ \\
\hline União estável & 741 & 692 & 93,4 & 49 & 6,6 & \\
\hline Solteira/separada/viúva & 306 & 259 & 84,6 & 47 & 15,4 & \\
\hline \multicolumn{7}{|l|}{ Escolaridade } \\
\hline Superior/médio completo & 374 & 358 & 95,7 & 16 & 4,3 & $<0,001$ \\
\hline Médio incompleto/fundamental completo & 390 & 351 & 90 & 39 & 10 & \\
\hline Fundamental completo/incompleto & 342 & 314 & 91,8 & 28 & 8,2 & \\
\hline Fundamental incompleto/analfabeto & 100 & 78 & 78 & 22 & 22 & \\
\hline \multicolumn{7}{|l|}{ Classe econômica ${ }^{b}$} \\
\hline$B 1^{c}+B 2^{d}+C 1^{e}$ & 301 & 301 & 92,3 & 25 & 7,7 & 0,096 \\
\hline$C 2^{f}$ & 482 & 482 & 92,5 & 39 & 7,5 & \\
\hline$D^{g}+E^{h}$ & 318 & 318 & 88,6 & 41 & 11,4 & \\
\hline \multicolumn{7}{|l|}{ Idade $1^{\text {a }}$ relação sexual (anos) } \\
\hline$\geq 20$ & 120 & 113 & 94,2 & 7 & 5,8 & 0,036 \\
\hline $19-15$ & 649 & 601 & 92,6 & 48 & 7,4 & \\
\hline$\leq 14$ & 437 & 387 & 88,6 & 50 & 11,4 & \\
\hline
\end{tabular}

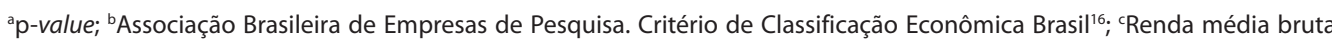

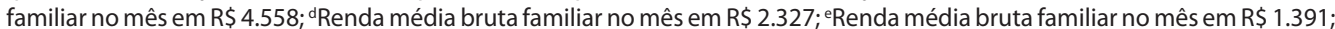
${ }^{f}$ Renda média bruta familiar no mês em R\$ 933; ${ }^{9}$ Renda média bruta familiar no mês em R\$ 618; ${ }^{\text {h }}$ Renda média bruta familiar no mês em R\$ 403; n: tamanho da amostra 
Tabela 1. Continuação...

\begin{tabular}{|c|c|c|c|c|c|c|}
\hline \multirow{3}{*}{ Características } & \multirow{3}{*}{$\begin{array}{c}\text { Total } \\
\mathrm{n}=1.206\end{array}$} & \multicolumn{4}{|c|}{ Pré-natal } & \multirow{3}{*}{ Valor $p^{a}$} \\
\hline & & \multicolumn{2}{|c|}{$\operatorname{Sim}(91,3 \%)$} & \multicolumn{2}{|c|}{ Não $(8,7 \%)$} & \\
\hline & & $\mathrm{n}=1.101$ & $\%$ & $n=105$ & $\%$ & \\
\hline \multicolumn{7}{|l|}{ Idade $1^{\text {a }}$ gravidez (anos) } \\
\hline$\geq 20$ & 443 & 419 & 94,6 & 24 & 5,4 & 0,003 \\
\hline $19-15$ & 661 & 595 & 90 & 66 & 10 & \\
\hline$\leq 14$ & 102 & 87 & 85,3 & 15 & 14,7 & \\
\hline \multicolumn{7}{|c|}{ Número de gestações anteriores } \\
\hline 1 & 447 & 422 & 94,4 & 25 & 5,6 & 0,003 \\
\hline $2-3$ & 521 & 473 & 90,8 & 48 & 9,2 & \\
\hline$\geq 4$ & 238 & 206 & 86,6 & 32 & 13,4 & \\
\hline \multicolumn{7}{|l|}{ Número de parceiros sexuais } \\
\hline 1 & 970 & 908 & 93,6 & 62 & 6,4 & $<0,001$ \\
\hline 2 & 141 & 120 & 85,1 & 21 & 14,9 & \\
\hline$\geq 3$ & 95 & 73 & 76,8 & 22 & 23,2 & \\
\hline \multicolumn{7}{|l|}{ Aborto anterior } \\
\hline Não & 875 & 827 & 94,5 & 48 & 5,5 & $<0,001$ \\
\hline Sim & 331 & 274 & 82,8 & 57 & 17,2 & \\
\hline
\end{tabular}

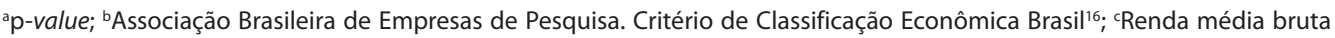

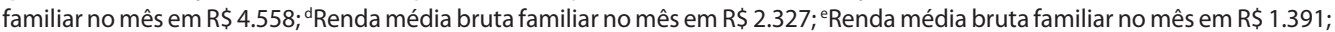
'Renda média bruta familiar no mês em R\$ 933; 'Renda média bruta familiar no mês em R\$ 618; 'Renda média bruta familiar no mês em R\$ 403; n: tamanho da amostra

Tabela 2. Características demográficas e da assistência entre mulheres, segundo o registro do VDRLa no cartão do pré-natal/prontuário. Recife, Pernambuco, Brasil, 2013-2014

\begin{tabular}{|c|c|c|c|c|c|c|}
\hline \multirow{3}{*}{ Características } & \multirow{3}{*}{$\begin{array}{c}\text { Total } \\
n=1.101\end{array}$} & \multicolumn{4}{|c|}{ VDRLa no cartão pré-natal/prontuário } & \multirow{3}{*}{ Valor $\mathbf{p}^{c}$} \\
\hline & & \multicolumn{2}{|c|}{ Registrado $^{b}(76,1 \%)$} & \multicolumn{2}{|c|}{ Não registrado $(23,9 \%)$} & \\
\hline & & $n=838$ & $\%$ & $n=263$ & $\%$ & \\
\hline \multicolumn{7}{|l|}{ Local de residência } \\
\hline Recife & 511 & 388 & 75,9 & 123 & 24,1 & 0,426 \\
\hline Região Metropolitana do Recife & 360 & 268 & 74,4 & 92 & 25,6 & \\
\hline Outros municípios & 230 & 182 & 79,1 & 48 & 20,9 & \\
\hline \multicolumn{7}{|l|}{ Idade (anos) } \\
\hline$\geq 35$ & 111 & 90 & 81,1 & 21 & 18,9 & 0,241 \\
\hline 34 a 20 & 701 & 523 & 74,6 & 178 & 25,4 & \\
\hline 19 a 12 & 289 & 225 & 77,9 & 64 & 22,1 & \\
\hline
\end{tabular}

aVDRL: Veneral Disease Research Laboratory; ${ }^{\text {In }}$ clui os registros do exame de sífilis durante o pré-natal sob forma qualitativa ou quantitativa no cartão do pré-natal, ficha de admissão ou prontuário; 'p-value; dPSF: Programa Saúde da Família; n: tamanho da amostra 
Tabela 2. Continuação...

\begin{tabular}{|c|c|c|c|c|c|c|}
\hline \multirow{3}{*}{ Características } & \multirow{3}{*}{$\begin{array}{c}\text { Total } \\
n=1.101\end{array}$} & \multicolumn{4}{|c|}{ VDRLa no cartão pré-natal/prontuário } & \multirow{3}{*}{ Valor $\mathbf{p}^{c}$} \\
\hline & & \multicolumn{2}{|c|}{ Registrado $^{b}(76,1 \%)$} & \multicolumn{2}{|c|}{ Não registrado $(23,9 \%)$} & \\
\hline & & $n=838$ & $\%$ & $\mathrm{n}=\mathbf{2 6 3}$ & $\%$ & \\
\hline \multicolumn{7}{|c|}{ Início pré-natal (em trimestre) } \\
\hline 10 & 758 & 601 & 79,3 & 157 & 20,7 & $<0,001$ \\
\hline 20 & 308 & 22 & 72,1 & 86 & 27,9 & \\
\hline 30 & 35 & 15 & 42,9 & 20 & 57,1 & \\
\hline \multicolumn{7}{|l|}{ Local pré-natal } \\
\hline $\mathrm{PSF}^{\mathrm{d}}$ & 710 & 534 & 75,2 & 176 & 24,8 & 0,016 \\
\hline Ambulatório / hospital & 256 & 210 & 82 & 46 & 18 & \\
\hline Outros & 135 & 94 & 69,6 & 41 & 30,4 & \\
\hline \multicolumn{7}{|l|}{ Consultas pré-natal } \\
\hline$\geq 6$ & 671 & 575 & 85,7 & 96 & 14,3 & $<0,001$ \\
\hline $5-4$ & 227 & 177 & 78 & 50 & 22 & \\
\hline $3-1$ & 203 & 86 & 42,4 & 117 & 57,6 & \\
\hline \multicolumn{7}{|l|}{ Categoria profissional } \\
\hline Enfermeiro & 623 & 469 & 75,3 & 154 & 24,7 & 0,55 \\
\hline Médico & 478 & 369 & 77,2 & 109 & 22,8 & \\
\hline \multicolumn{7}{|c|}{ Solicitado exames na $1^{a}$ consulta } \\
\hline Sim & 1044 & 807 & 77,3 & 237 & 22,7 & $<0,001$ \\
\hline Não & 57 & 31 & 54,4 & 26 & 45,6 & \\
\hline \multicolumn{7}{|c|}{ Entrega dos resultados (em dias) } \\
\hline $0-15$ & 702 & 577 & 82,2 & 125 & 17,8 & $<0,001$ \\
\hline $16-30$ & 246 & 200 & 81,3 & 46 & 18,7 & \\
\hline$\geq 31$ & 153 & 61 & 39,9 & 92 & 60,1 & \\
\hline
\end{tabular}

aVDRL: Veneral Disease Research Laboratory; 'bnclui os registros do exame de sífilis durante o pré-natal sob forma qualitativa ou quantitativa no cartão do pré-natal, ficha de admissão ou prontuário; ${ }^{\complement} p$-value; dPSF: Programa Saúde da Família n: tamanho da amostra

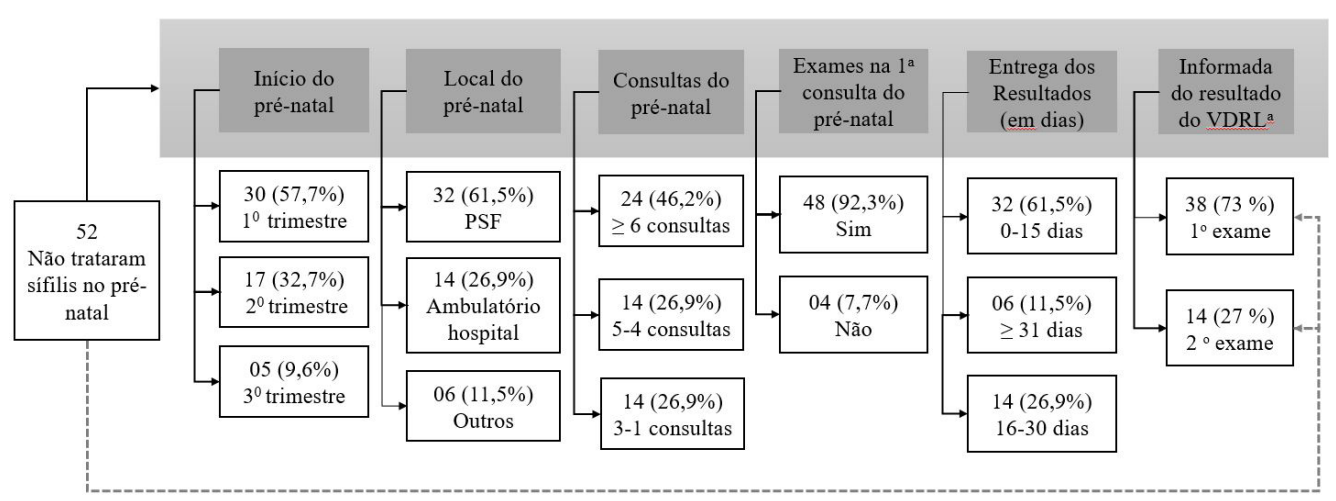

Figura 1. Características assistenciais das mulheres que não realizaram tratamento para sífilis durante o pré-natal. Recife, Pernambuco, Brasil, 2013-2014. aVDRL: Veneral Disease Research Laboratory 


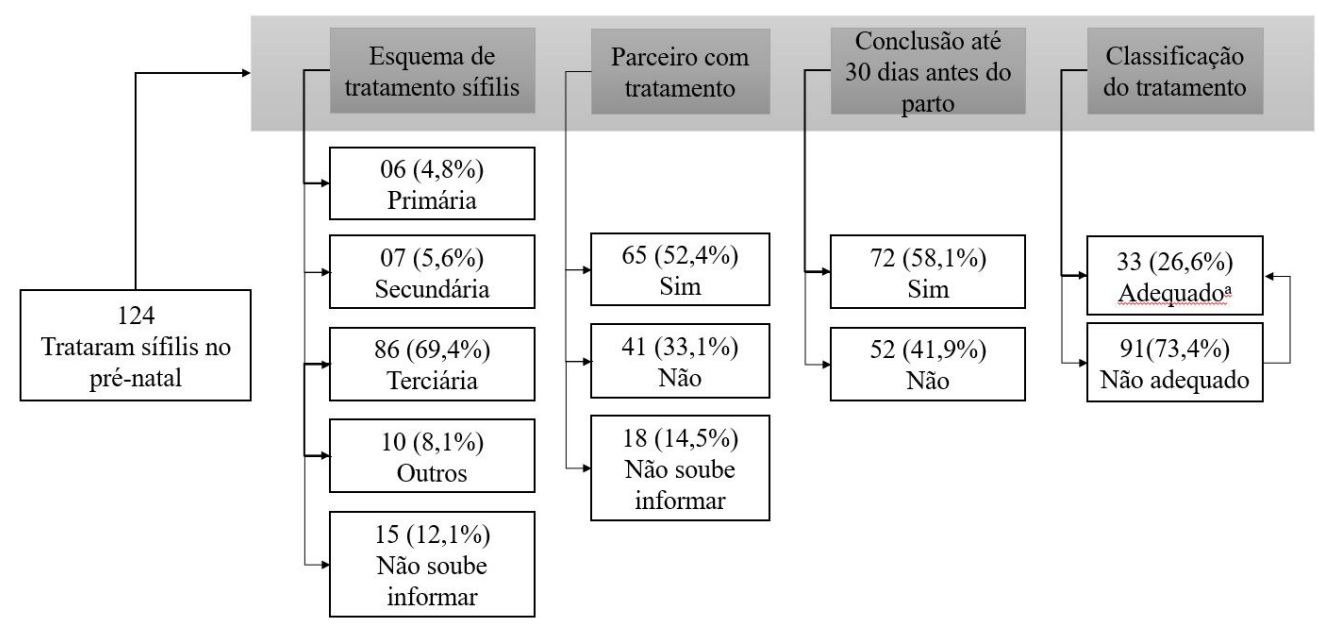

Figura 2. Manejo de casos em mulheres que relataram a realização do tratamento para sífilis durante

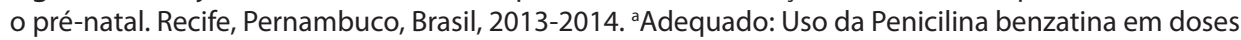
preconizadas pelo Ministério da Saúde; ter finalizado o tratamento até 30 dias antes do parto; parceiro tratado concomitantemente com o mesmo esquema de tratamento; registro no cartão do pré-natal e, na sua ausência, o relato da mulher

Entre os principais motivos referidos pelas gestantes para a não realização do tratamento (27\%) foi o conhecimento do VDRL reagente apenas no segundo exame e com curto intervalo até a admissão na maternidade. Destas, três mulheres sabiam dos resultados do VDRL no momento da entrevista, mas não realizaram o acompanhamento pré-natal. Dentre as gestantes com VDRL reagente na admissão, 62,9\% não realizaram pré-natal, enquanto $46,3 \%$ o fizeram, mas não tinham o registro do exame no cartão (dados não tabulados).

Entre as mulheres que fizeram um dos exames de VDRL durante o pré-natal, 176 souberam do diagnóstico de sífilis durante a gestação, destas, $124(70,5 \%)$ trataram a sífilis na gestação atual, $69,4 \%$ receberam a dose da penicilina benzatina para sífilis terciária e 8,1\% foram medicadas sem a sua utilização. Apenas $52,4 \%$ das mulheres informaram que seus parceiros receberam tratamento concomitante, e 58,1\% concluíram o tratamento até 30 dias antes do parto. Observou-se que $73,4 \%$ receberam tratamento classificado como não adequado para sífilis (Figura 2).

\section{DISCUSSÃO}

Os resultados mostraram que as gestantes atendidas e investigadas nas maternidades do Recife enfrentaram barreiras de acesso ao pré-natal em aspectos relacionados à transmissão vertical da sífilis, caracterizando situações iníquas, inclusive as assistenciais, que são expressão das desigualdades e diferentes oportunidades de atenção à saúde aos grupos vulneráveis 15,17 $^{10}$.

Apesar dos investimentos na melhoria da atenção à saúde das mulheres nas últimas décadas, permanecem obstáculos na assistência pré-natal e ao parto que repercutem no alcance de metas pactuadas e resultam na inadequação do cuidado ${ }^{13,18}$. A ausência do pré-natal identificada em $8,7 \%$ das gestantes esteve fortemente associada às condições de vida e comportamentais, semelhante a outros achados ${ }^{10,19}$. A privação do cuidado pré-natal é extensamente documentada como amplificadora de efeitos adversos na gestação e parto entre mulheres e recém-nascidos, a exemplo de sífilis congênita, aborto, natimortalidade, morte neonatal e prematuridade $1,6,8$.

Entre as mulheres que declararam ter realizado o pré-natal, identificou-se de forma variada, porém ainda expressiva, o início tardio da assistência, número inadequado de consultas, não solicitação dos exames na primeira consulta e elevado tempo de entrega dos resultados, que podem explicar, em parte, a permanência de desfechos negativos, como a transmissão vertical da sífilis. Estudos nacionais e locais sobre a avaliação do pré-natal demonstram que os avanços 
na assistência perinatal se alternam com falhas, resultando em baixa adequação e predomínio de ações parcialmente implantadas ${ }^{13,20}$.

A despeito do elevado registro do VDRL no cartão (76,1\%), alguns estudos mostraram discrepâncias entre as anotações no cartão e o informado pela gestante ${ }^{21-23}$, dificultando a comunicação entre níveis assistenciais de saúde. $O$ adequado registro pode expressar os mecanismos de coordenação do cuidado e a adesão das categorias profissionais que atuam na assistência pré-natal ${ }^{24,25}$.

A análise do perfil das gestantes sem a transcrição do VDRL no cartão possibilita melhor compreensão sobre a qualidade do acompanhamento e os cuidados efetivamente fornecidos a esse grupo, sendo marcantes as diferenças, em relação às com anotação do VDRL no cartão 26,27 . As gestantes sem registro do VDRL apresentaram maior frequência do início do pré-natal no último trimestre, baixo número de consultas e ausência de solicitação e entrega tardia dos resultados de exames.

Igualmente importantes são as diferenças entre os grupos quanto à ausência do registro ou não realização do segundo exame VDRL. Na ocasião da admissão da maternidade verificou-se que, entre as sem a transcrição do VDRL, 106 (40,3\%) apresentavam resultado reagente, sendo que 51 (19,4\%) com titulação superior a 1:8 (dados não tabulados).

Os protocolos nacionais recomendam a realização de dois VDRL durante a gestação. A não realização do exame e a ausência da entrega do resultado no prazo de 15 dias se incluem entre as condições que mais se relacionam com falhas e inadequação da assistência pré-natal ${ }^{13,28}$. O estudo "parturiente sentinela" mostrou que apenas $16,9 \%$ das gestantes apresentaram o registro dos dois testes conforme preconizado 27.

As características assistenciais de gestantes quanto à realização da sífilis no pré-natal sinalizam para uma vigilância e assistência deficiente, com perda de oportunidades de prevenção e tratamento da sífilis congênita. De forma similar a outros estudos ${ }^{11,20,29}$, grande parte das gestantes realizou o acompanhamento na estratégia saúde da família e apresentou número adequado de consultas, com solicitação e entrega dos exames em tempo hábil.

Além das barreiras de acesso ao pré-natal, existem as associadas ao baixo conhecimento dos protocolos assistenciais e dificuldades na abordagem das infecções sexualmente transmissíveis pelos profissionais de saúde, aspecto relatado ao se avaliar o manejo da sífilis entre pré-natalistas ${ }^{20,29}$. Adicionalmente, não se identificou associação no acompanhamento à gestante e o controle da sífilis congênita entre a estratégia saúde da família e outros modelos de atenção' ${ }^{29}$.

A ocorrência de qualquer evento adverso da gravidez é $52 \%$ maior entre as mulheres com sífilis ${ }^{30}$, em decorrência recomenda-se reorganizar os fluxos e ações assistenciais, com integração de programas materno-infantil locais, busca ativa das gestantes e parceiros sem atendimento e tratamento, visitas domiciliares, ampliação do planejamento familiar e a integração entre a vigilância, a assistência à saúde e a intersetorialidade, priorizando mulheres de risco 18,19,31,32.

Em relação à realização do tratamento para sífilis na gestação, os achados refletem a insuficiente adesão às normas assistenciais para triagem e tratamento, pouco envolvimento e preparo da equipe de saúde diante de um resultado reagente, dificuldade de captação dos parceiros e elevada proporção de tratamento ineficaz, o que repercutiu na baixa proporção de tratamentos adequados, similar a outros estudos ${ }^{19,33}$. As inadequações no tratamento das infecções sexualmente transmissíveis são relatadas tanto na atenção primária quanto em outros níveis assistenciais ao identificar gestantes sem conhecimento dos resultados sorológicos de sífilis e HIV e sobre a importância do tratamento de seus parceiros ${ }^{34,35}$.

Entre as limitações da pesquisa, destaca-se a utilização de banco de dados oriundo de um estudo caso-controle, cujos resultados podem apresentar vieses de seleção e informação, que foram minimizados ao comparar as barreiras demográficas, sociais e assistenciais entre gestantes, segundo condição de realização do pré-natal. Igualmente, a incompletude de registros nos cartões de pré-natal ou sua ausência ocasionou a utilização da informação da entrevistada, podendo ter comprometido a fidedignidade dos achados.

Observaram-se diferentes obstáculos para diagnosticar precocemente, tratar oportunamente e adequadamente a sífilis durante o pré-natal. Os resultados do VDRL na 
admissão à maternidade apresentaram importantes diferenças entre gestantes segundo situação de realização do pré-natal, que dificultaram o diagnóstico e o tratamento adequado, favorecendo a transmissão vertical da sífilis. Entre as que não realizaram o pré-natal, ocorreram percentuais expressivos da infecção, possibilitando aumento de desfechos perinatais negativos. E mesmo entre as gestantes que realizaram o pré-natal houve mulheres reagentes para sífilis, ainda que em menor proporção, sendo perdida a oportunidade de alcançar o controle da doença.

A avaliação permitiu identificar situações que expressam as desiguais oportunidades de atenção à saúde de grupos vulneráveis e distintas barreiras assistenciais no pré-natal que retardaram o diagnóstico e, consequentemente, o tratamento apropriado para a sífilis.

A ampliação do acesso ao diagnóstico, favorecendo o início precoce do pré-natal, melhor organização dos fluxos assistenciais nos serviços, integrando os diferentes níveis do cuidado, revela-se como uma prioridade a ser perseguida. A identificação das mulheres que estão expostas a fatores de risco favorece a implementação de estratégias para o ingresso na assistência. São alguns exemplos de ações imediatas que podem provocar uma abordagem sustentável de médio a longo prazo e, assim, contribuir de forma mais efetiva no controle da sífilis no país.

\section{REFERÊNCIAS}

1. Cerqueira LRP, Monteiro DLM, Taquette SR, Rodrigues NCP, Trajano AJB, Rodrigues NCP, et al. The magnitude of syphilis: from prevalence to vertical transmission. Rev. Inst. Med. trop. S. Paulo. 2017;59(e78):1-7.

2. Domingues RM, Szwarcwald CL, Souza PR Jr, Leal MC. Prevalence of syphilis in pregnancy and prenatal syphilis testing in Brazil: Birth in Brazil study. Rev Saude Publica. 2014;48(5):766-74. http://dx.doi. org/10.1590/S0034-8910.2014048005114. PMid:25372167.

3. Brasil. Ministério da Saúde. Secretaria de Vigilância em Saúde. Departamento de Vigilância, Prevenção e Controle das Infecções Sexualmente Transmissíveis, do HIV/Aids e das Hepatites Virais (DIAHV). Sífilis 2018. Boletim Epidemiológico. 2018;49(45):1-43.

4. Serruya SJ, Duran P, Martinez G, Romero M, Caffe S, Alonso M, et al. Maternal and congenital syphilis in selected Latin America and Caribbean countries: a multi-country analysis using data from the Perinatal Information System. Sex Health. 2015;12(2):164-9. http://dx.doi.org/10.1071/SH14191. PMid:25725563.

5. Saraceni V, Pereira GFM, Silveira MF, Araujo MAL, Miranda AE. Vigilância epidemiológica da transmissão vertical da sífilis: dados de seis unidades federativas no Brasil. Rev Panam Salud Publica. 2017;41:e44. PMid:28614467.

6. Domingues RMSM, Leal MC. Incidência de sífilis congênita e fatores associados à transmissão vertical da sífilis: dados do estudo Nascer no Brasil. Cad Saude Publica. 2016;32(6). http://dx.doi.org/10.1590/0102311X00082415. PMid:27333146.

7. Casal CA, Silva MO, Costa IB, Araújo EC, Corvelo TC. Molecular detection of Treponema pallidum sp. pallidum in blood samples of VDRL-seroreactive women with lethal pregnancy outcomes: a retrospective observational study in northern Brazil. Rev Soc Bras Med Trop. 2011;44(4):451-6. http://dx.doi.org/10.1590/ S0037-86822011005000047. PMid:21789353.

8. Magalhães DMS, Kawaguchi IAL, Dias A, Calderon IMP. Sífilis materna e congênita: ainda um desafio. Cad Saude Publica. 2013;29(6):1109-20. http://dx.doi.org/10.1590/S0102-311X2013000600008. PMid:23778543.

9. Araújo MAL, Barros VL, Moura HJ, Rocha AFB, Guanabara MAO. Prevenção da sífilis congênita em Fortaleza, Ceará: uma avaliação de estrutura e processo. Cad Saude Colet. 2014;22(3):300-6. http://dx.doi. org/10.1590/1414-462X201400030012.

10. Viellas EF, Domingues RMSM, Dias MAB, Gama SGN, Theme MM Fa, Costa JV, et al. Assistência pré-natal no Brasil. Cad Saude Publica. 2014 ago;30(Suppl 1):S1-15. http://dx.doi.org/10.1590/0102-311X00126013. PMid:25167194.

11. Domingues RMSM, Leal MC, Hartz ZMA, Dias MAB, Vettore MV. Access to and utilization of prenatal care services in the Unified Health System of the city of Rio de Janeiro, Brazil. Rev Bras Epidemiol. 2013;16(4):95365. http://dx.doi.org/10.1590/S1415-790X2013000400015. PMid:24896600.

12. Leal MC, Theme-Filha MM, Moura EC, Cecatti JG, Santos LMP. Atenção ao pré-natal e parto em mulheres usuárias do sistema público de saúde residentes na Amazônia Legal e no Nordeste, Brasil 2010. Rev Bras Saúde Mater Infant. 2015;15(1):91-104. http://dx.doi.org/10.1590/S1519-38292015000100008. 
13. Xavier RB, Jannotti CB, Silva KS, Martins AC. Risco reprodutivo e renda familiar: análise do perfil de gestantes. Cien Saude Colet. 2013;18(4):1161-71. http://dx.doi.org/10.1590/S1413-81232013000400029. PMid:23670393.

14. Rosa CQ, Silveira DS, Costa JS. Factors associated with lack of prenatal care in a large municipality. Rev Saude Publica. 2014;48(6):977-84. http://dx.doi.org/10.1590/S0034-8910.2014048005283. PMid:26039401.

15. Macêdo VC, Lira PIC, Frias PG, Romaguera LMD, Caires SFF, Ximenes RAA. Risk factors for syphilis in women: case-control study. Rev Saude Publica. 2017;51:78. http://dx.doi.org/10.11606/s15188787.2017051007066. PMid:28832758.

16. Associação Brasileira de Empresa de Pesquisa. Critério de Classificação Econômica Brasil 2009. São Paulo: ABEP; 2009. Disponível em: http://www.abep.org/criterio-brasil

17. Gama SGN, Szwarcwald CL, Sabroza AR, Castelo Branco V, Leal MC. Fatores associados a assistência pré-natal precária em uma amostra de puérperas adolescentes em maternidades do município do Rio de Janeiro, 1999-2000. Cad Saude Publica. 2004;20(Suppl 1):101-11. http://dx.doi.org/10.1590/S0102311X2004000700011. PMid:16636740.

18. Brasil. Ministério da Saúde. Secretaria de Vigilância em Saúde. Departamento de Vigilância, Prevenção e Controle das Infecções Sexualmente Transmissíveis, do HIV/Aids e das Hepatites Virais. Agenda de Ações Estratégicas para Redução da Sífilis no Brasil. Brasília: Ministério da Saúde; 2017.

19. Cavalcante MGS, Samico I, Frias PG, Vidal SA. Análise de implantação das áreas estratégicas da atenção básica nas equipes de Saúde da Família em município de uma Região Metropolitana do Nordeste Brasileiro. Rev Bras Saúde Mater Infant. 2006;6(4):437-45. http://dx.doi.org/10.1590/S1519-38292006000400011.

20. Domingues RMSM, Viellas EF, Dias MAB, Torres JA, Theme-Filha MM, Gama SGN, et al. Adequação da assistência pré-natal segundo as características maternas no Brasil. Rev Panam Salud Publica. 2015;37(3):140-7. PMid:25988250.

21. Santos ET No, Leal MC, Oliveira AE, Zandonade E, Gama SGN. Concordância entre informações do Cartão da Gestante e da memória materna sobre assistência pré-natal. Cad Saude Publica. 2012;28(2):256-66. http://dx.doi.org/10.1590/S0102-311X2012000200005. PMid:22331152.

22. Santos ET No, Oliveira AE, Zandonade E, Gama SGN, Leal MC. O que os cartões de pré-natal das gestantes revelam sobre a assistência nos serviços do SUS da Região Metropolitana da Grande Vitória, Espírito Santo, Brasil? Cad Saude Publica. 2012;28(9):1650-62. http://dx.doi.org/10.1590/S0102-311X2012000900005. PMid:23033181.

23. Barreto FD, Albuquerque RM. Discrepâncias entre o informe verbal e os registros no cartão da gestante, um instrumento negligenciado. Rev Bras Ginecol Obstet. 2012;34(6):259-67. http://dx.doi.org/10.1590/ S0100-72032012000600004. PMid:22801600.

24. Saraceni V, Miranda AE. Relação entre a cobertura da estratégia saúde da família e o diagnóstico de sífilis na gestação e sífilis congênita. Cad Saude Publica. 2012;28(3):490-6. http://dx.doi.org/10.1590/S0102311X2012000300009. PMid:22415181.

25. Vázquez ML, Vargas I, Unger JP, De Paepe P, Mogollón-Pérez AS, Samico I, et al. Evaluating the effectiveness of care integration strategies in different healthcare systems in Latin America: the EQUITYLA II quasi-experimental study protocol. BMJ Open. 2015;5(7):e007037. http://dx.doi.org/10.1136/ bmjopen-2014-007037. PMid:26231753.

26. Polgliani RBS, Santos ET No, Zandonade E. Informações dos cartões de gestantes e dos prontuários da atenção básica sobre assistência pré-natal. Rev Bras Ginecol Obstet. 2014;36(6):269-75. http://dx.doi. org/10.1590/S0100-720320140004907. PMid:25099467.

27. Szwarcwald CL, Barbosa A Jr, Miranda AE, Paz LC. Results of the sentinel surveillance project, 2006: challenges for the control of congenital syphilis in Brazil. DST J Bras Doenças Sex Transm. 2007;19:128-33.

28. Oliveira Guanabara MA, Leite-Araújo MA, Matsue RY, Lima de Barros V, Alves Oliveira F. Acesso de gestantes às tecnologias para prevenção e controle da sífilis congênita em Fortaleza-Ceará, Brasil. Rev Salud Publica (Bogota). 2017;19(1):73-8. http://dx.doi.org/10.15446/rsap.v19n1.49295. PMid:30137158.

29. Araújo CL, Shimizu HE, Sousa AIA, Hamann EM. Incidence of congenital syphilis in Brazil and its relationship with the Family Health Strategy. Rev Saude Publica. 2012;46(3):479-86. http://dx.doi.org/10.1590/S003489102012000300010. PMid:22635036.

30. Gomez GB, Kamb ML, Newman LM, Mark J, Broutet N, Hawkes SJ. Untreated maternal syphilis and adverse outcomes of pregnancy: a systematic review and meta-analysis. Bull World Health Organ. 2013;91(3):21726. http://dx.doi.org/10.2471/BLT.12.107623. PMid:23476094.

31. Vidal SA, Samico IC, Frias PG, Hartz ZMA. Estudo exploratório de custos e conseqüências do pré-natal no Programa Saúde da Família. Rev Saude Publica. 2011 jun;45(3):467-74. http://dx.doi.org/10.1590/S003489102011005000014. PMid:21445460. 
32. Brasil. Ministério da Saúde. Comissão Nacional de Incorporação de Tecnologias no SUS. Penicilina benzatina para prevenção da Sífilis Congênita durante a gravidez. Brasília: Ministério da Saúde; 2015

33. Domingues RMSM, Lauria LM, Saraceni V, Leal MC. Manejo da sífilis na gestação: conhecimentos, práticas e atitudes dos profissionais pré-natalistas da rede SUS do município do Rio de Janeiro. Ciênc. Saúde Coletiva. 2013;18(5):1341-51. http://dx.doi.org/10.1590/S1413-81232013000500019.

34. Macêdo VC, Bezerra AFB, Frias PG, Andrade CLT. Avaliação das ações de prevenção da transmissão vertical do HIV e sífilis em maternidades públicas de quatro municípios do Nordeste brasileiro. Cad Saude Publica. 2009;25(8):1679-92. http://dx.doi.org/10.1590/S0102-311X2009000800004. PMid:19649409.

35. Campos ALA, Araújo MAL, Melo SP, Andrade RFV, Gonçalves MLC. Sífilis em parturientes: aspectos relacionados ao parceiro sexual. Rev Bras Ginecol Obstet. 2012;34(9):397-402. http://dx.doi.org/10.1590/ S0100-72032012000900002. PMid:23197277. 